\title{
Calibration Point for Electron Ionization MS / MS Spectra Measured with Multiquadrupole Mass Spectrometers
}

\author{
Krishnan R. Mohan, Michael G. Bartlett, and Kenneth L. Busch \\ School of Chemistry and Biochemistry, Georgia Institute of Technology, Atlanta, Georgia, USA
}

\author{
Alan E. Schoen and Nigel Gore \\ Finnigan MAT, 355 River Oaks Parkway, San Jose, California, USA
}

\begin{abstract}
A protocol for establishing standard instrument conditions for measurement of product ion MS/MS spectra from parent ions produced by electron ionization is presented. Within this protocol, the ion at $m / z 231\left(\mathrm{C}_{5} \mathrm{~F}_{9}^{+}\right)$from perfluorokerosene or perfluorotributylamine is selected as the parent ion and subjected to collision-induced dissociation. The relative intensities of product ions at $m / z \quad 69,131$, and 181 are monitored as a function of collision energy while keeping the target gas pressure constant within the range of $10^{-4}-10^{-6}$ torr (measured), or a beam attenuation of approximately 30-70\%. The collision energy at which the ion intensities for product ions at $m / z 69$ and 181 are equal is defined as the calibration point at that collision gas pressure; the intensity of the ion at $m / z 131$ is very close to this value as well. Electron ionization MS/MS spectra taken at the calibration point using two different multiquadrupole instruments show good reproducibility for several test compounds. The high degree of similarity may aid in the establishment of a MS/MS spectral library. (J Am Soc Màss Spectrom 1994, 5, 576-582)
\end{abstract}

$\mathrm{T}$ andem mass spectrometry (MS/MS) experiments can be performed on multisector, multiquadrupole, or hybrid instruments of different geometries. The latter two instruments use a radiofrequency ( $\mathrm{rf}$ )-only quadrupole as the collision cell. Collision-induced dissociation (CID) within triple quadrupole (QqQ) or hybrid (EBqQ or BEqQ) sector/quadrupole instruments usually involves low collision energies (10-100 eV), multiple collisions with the target gas, and extended ion residence times (up to several microseconds) in the dissociation region. A parent ion selected by the first quadrupole $(Q)$ or sectors (EB or $\mathrm{BE}$ ) interacts with a collision gas in the rf-only quadrupole collision cell (q). The product ions and undissociated parent ions pass into the final quadrupole (Q) for mass analysis. The instrument thus produces a CID mass spectrum of each initially selected parent ion.

In principle, standard CID MS/MS spectra could be generated, collected, and stored in reference libraries. These reference spectra could later be used for comparison with MS/MS spectra measured for unknown compounds in a manner analogous to the use of refer-

Address reprint requests to Kenneth L. Busch, School of Chemistry and Biochemistry, Georgia Institute of Technology, Atlanta, GA $30332-0400$. ence libraries of electron ionization (EI) mass spectra. Further, it should be possible to infer the identity of an unknown complex molecule by identifying the ionic substructures of fragment ions generated in its CID mass spectrum. I Iowever, to date, reference libraries of CID-tandem mass have not been collected because of a lack of standardization of operating conditions of different instruments. One notes specifically that this lack of standardization has not prevented the widespread use of quadrupole-based MS/MS for quantitative analysis, for ion structural determinations, and indeed for the measurement of fundamental thermochemical properties for a number of different systems.

Some means to acquire instrument-independent MS/MS spectra are a prerequisite for development of a common database similar to those available for EI mass spectra. However, there are several instrument parameters that can cause significantly different MS/MS product ion spectra to be observed for any given molecule. The key adjustable parameters are: (1) the number of collisions undergone by a parent ion within the second (rf-only) quadrupole, a parameter usually characterized in terms of the pressure of the collision gas, and (2) the energetic parameter describing the interaction between the parent ion and the collision gas, which is determined by the collision energy for parent ions entering the collision 
quadrupole. Other parameters can affect the measured MS/MS spectrum, but these are not usually manipulated in the experiment.

A 1982 collaborative study organized by Dawson and Sun [1] addressed the issue of MS/MS spectral reproducibility. The high level of interest provided the impetus for a round-robin test for spectral comparison of a few selected compounds to assess reproducibility and comparability of spectra generated on different multiquadrupole instruments. Very different MS/MS spectra were observed in various laboratories for $\mathbf{n}$ butylbenzene and protonated dimethylphthalate, the chosen model compounds. Assessment of the data emphasized that the reproducibility of MS/MS spectra depends on a number of instrumental factors, including collision gas pressure, collision energy, and rf tuning [2]. Moderately good agreement between various sets of data could be obtained, but it was also observed that instrument parameters (collision energy or collision cell pressure), as recorded from the instrument readouts, were not sufficiently reproducible to specify library-quality operating conditions for low collision energy MS/MS spectral measurement [2].

In a carefully constructed series of experiments, Martinez and Dheandhanoo [3, 4] have emphasized that the creation of MS/MS spectral libraries is contingent upon the development of instrument-independent operating conditions for the data collection. Several protocols [5-7] have been suggested for measurement of instrument-independent MS/MS spectra in quadrupole collision cells, including procedures based on the charge exchange reaction of argon, or the CID of the acetone cation. However, some of these suggested protocols can involve instrument parameters far removed from those relevant to day-to-day opcration. In that evaluations of instrument performance can also involve disabling of automated instrument control functions, there is also some reluctance to incorporate these into a daily operating protocol. Mirocha and Pawlosky [8] have suggested that standard MS/MS conditions may be achieved based upon CID of the tetramethylsilane derivative of zearalenone; results of this collaborative work were presented at a recent American Society for Mass Spectrometry conference.

The operation of an rf-only quadrupole for CID MS/MS experiments is far more complex than generally realized. Transmission of parent ions into the region, and transmission of product ions out of the rf-only quadrupole into the final mass analyzing quadrupole, both exhibit dependence on the ion kinetic energy established by the quadrupole potential float values relative to the potential of the ion source. Certainly, the physical size and shape of the quadrupole rods themselves, as well as the frequency and amplitude of the rf power applied to them, influence ion transmission through the rf-only quadrupole region. Energy-resolved MS/MS experiments involve monitoring changes in product ion abundance as the collision energy of the parent ion is varied by change of the rf-only quadrupole voltage offset. Substantial changes in spectral appearance can be observed, and often the changes in product ion intensity follow expected variations in ratios of the products of simple cleavages versus those derived from rearrangements $[9,10]$. However, the existence of nodes in ion transmission through rf-only quadrupoles for both parent and product ions can also cause variation in observed ion intensity with changes in collision energy; these changes can be substantial [11-16]. It is for all these reasons that a carefully designed instrument-independent operation procedure was first suggested, and why it is the ultimate goal in instrument calibration.

Simple methods are presented here for establishing standard instrument conditions for measurement of product ion MS/MS spectra in multipole collision cells using EI. It is not suggested that the rigor of this method matches that of more involved procedures, but we suggest that this approach might be made part of a daily instrument tuning process, especially since the protocol involves the mass calibration compounds that are already readily available. The degree of similarity of MS/MS spectra obtained under these standard conditions was established by measurement of mass spectra of a randomly selected group of organic compounds on separate instruments in different laboratories. The ability to reach the calibration point conditions was established over a course of several years with several instrument operators. Despite differences in physical construction and in electronic operating parameters of the instruments, this work has determined that a fairly high level of spectral similarity can be expected when different instruments use the same calibration conditions.

\section{Experimental}

Two multiquadrupole mass spectrometers were tuned to a standard set of MS/MS conditions using the calibration point described below, and product ion MS/MS spectra were recorded for several different compounds under these standard conditions. A VGTOSEQ (Manchester, UK) hybrid mass spectrometer of EBqQ geometry ( $q$ is the collision quadrupole and $Q$ is the final mass-analyzing quadrupole) with standard EI, chemical ionization, and fast-atom bombardment ionization sources was used to measure MS/MS spectra in one laboratory. The EI conditions were $70-\mathrm{eV}$ electron energy and a trap current of $100 \mu \mathrm{A}$. The instrument was operated at a resolution of approximately 1500 ( $10 \%$ valley definition). At this laboratory, perfluorokerosene (PFK) was introduced into the source from the reservoir inlet system. Air was used as the collision gas in the hybrid instrument for MS/MS experiments. Indicated pressures on the ionization gauge nearest to the collision cell were in the range of $10^{-5}-10^{-6}$ torr. The actual collision gas pressure within the cell is certainly higher, and can be estimated [17, 
18] as a few millitorr. Since the calibration point is defined through variation of the collision energy, as described shortly, the common inability to accurately measure collision gas pressure is not pertinent here. The rf amplitude was not retuned between experiments, nor was any stipulation placed on rf amplitude from instrument to instrument. The hybrid instrument was set up initially by tuning the rf-only collision cell to a maximum transmission node for the precursor ion at $m / z 231$ from PFK [19]. The tuning parameters
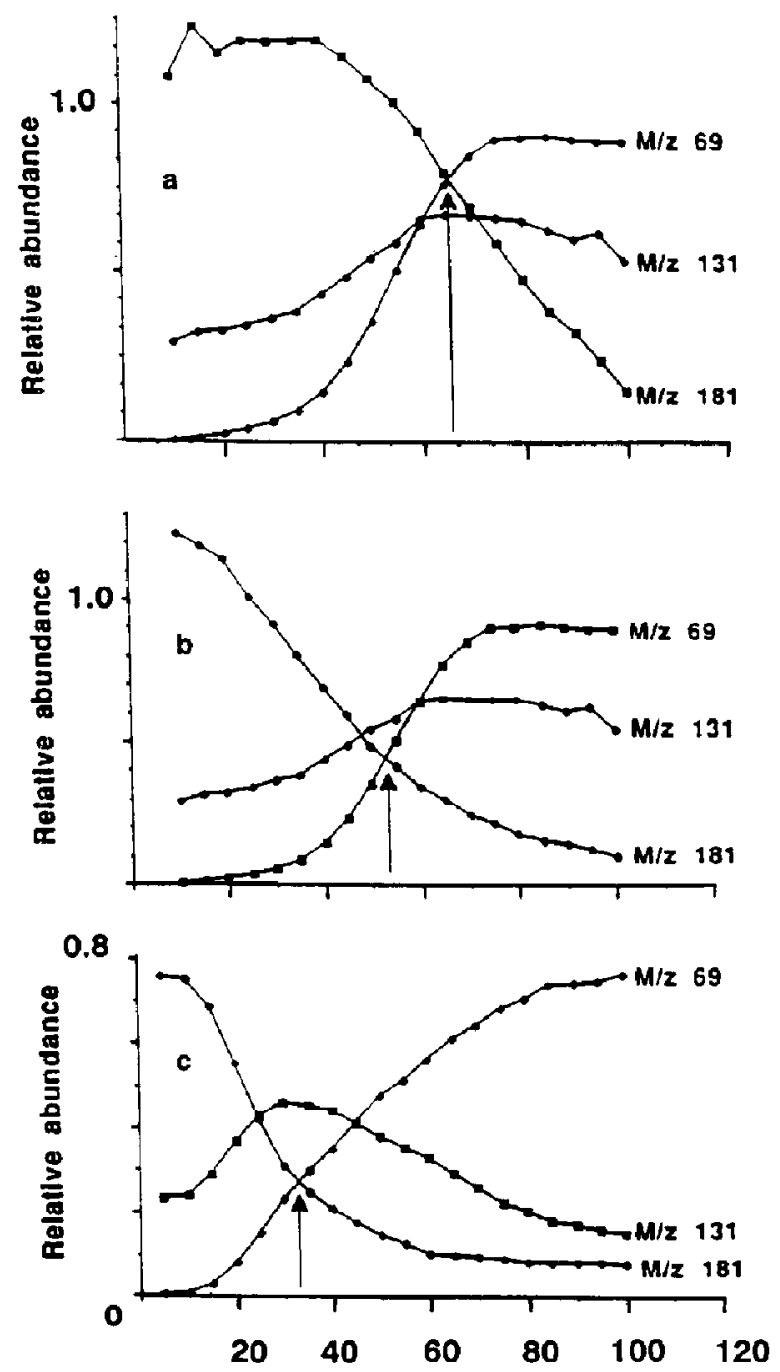

\section{Collision energy (eV)}

Figure 1. Variation of calibration point with collision gas pressure at three pressures $\left(3.75 \times 10^{-6}\right.$ torr (a), $7.5 \times 10^{-6}$ torr (b), and $3.75 \times 10^{-5}$ torr (c)) across the collision energy range of interest. The $x$ axis is collision energy in volts. Data were recorded on the hybrid geometry MS/MS instrument with PFK as the sample. remained here for the rest of the study. In measurement of MS/MS spectra at different collision energies, the system was set to the low collision energy, and the collision gas pressure increased until a parent ion beam attenuation of about $60 \%$ was reached. The collision energy was increased until the intensities of the ion beams at $m / z 69$ and $m / z 181$ were equal, and this was designated as the calibration point. The calibration point is therefore based empirically on the measurement of equal intensities for the two specified ions. At any specified tuning of the instrument, both (1) collision energy and (2) collision gas pressure are factored into target thickness, which then determines the (3) intensities of ions in the MS/MS spectrum. Specification of any two parameters determines the third. The first variable that is specified is the requirement of equal ion intensity. The second variable is the collision energy, easily accessed and accurately measured, which is then varied at any constant gas pressure to reach the point of equal ion intensities for the ions at $m / z 69$ and $m / z$ 181. The collision gas pressure must be stable, but is not an independent variable, and need not be accurately measured or sperified. Selection of equal product ion intensities at a specified collision energy was chosen as a more direct route to a calibration point than specification of a parent ion beam attenuation. This latter value also reflects many processes (ion transmission, scattering, neutralization) separate in focus from the creation of CID products.

A Finnigan MAT TSQ-700 (San Jose, CA) "triple quadrupole" mass spectrometer was used at the second laboratory. This instrument is equipped with an octapole collision cell; since these collision cells do not exhibit transmission nodes as do the quadrupolar cells, the comparison presented in this paper is particularly

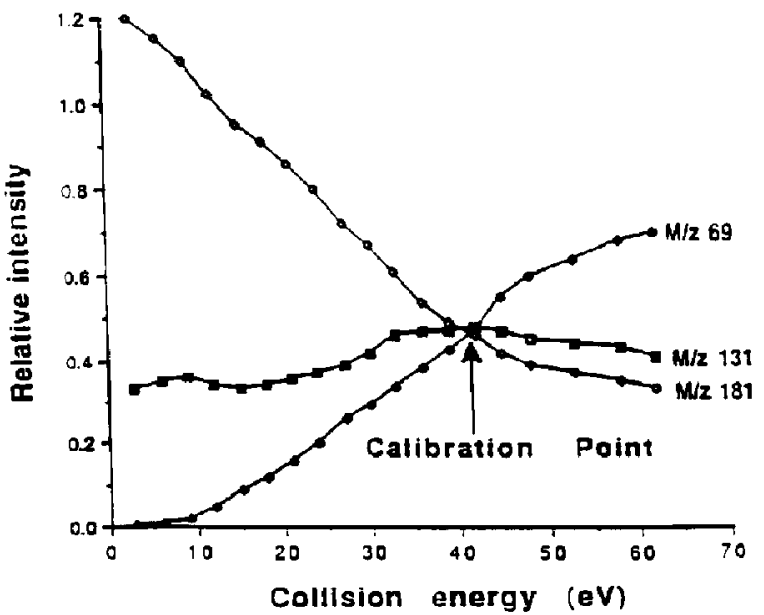

Figure 2. Calibration point plot determined for the Finnigan triple quadrupole MS/MS instrument with PFTBA as the sample. The $x$ axis is collision energy in volts. Pressure indicated for the collision cell by the thermocouple is about $1 \times 10^{-3}$ torr. 
convincing. The EI source was operated under standard conditions of $70-\mathrm{eV}$ electron energy and $200-\mu \mathrm{A}$ emission current. Perfluorotributylamine (PFTBA) was used here for both mass and EI-MS/MS calibration, and was leaked into the source until a stable and intense ion current for the ion at $m / z 231$ is attained. The collision gas (air) in the rf-only octapole was introduced to a pressure of around 1 mtorr as measured on a thermocouple gauge. The standard MS/MS operation point was then achieved through variance of the collision energy as described below. Samples analyzed in common at the two laboratories were obtained commercially, and divided from a common lot. The selection of model compounds was made at random from a selection of organic compounds of different classes available in the laboratory. This random selection was made to provide a realistic assessment of the quality of the general agreement that could be expected from laboratory to laboratory.

\section{Results and Discussion}

PFK and PFTBA are in common use as a mass calibration compound and are easily introduced into standard ionization sources. A high intensity, stable ion current was obtained for the ion at $m / z 231\left(\mathrm{C}_{5} \mathrm{~F}_{9}^{+}\right)$, which was then selected as the parent ion and subjected to CID. Product ions at $m / z$ 181, 131, and 69 were obtained by losses of $C_{2}, C_{2} F_{4}$, and $C_{4} F_{6}$, respectively, from the parent ion. The relative intensities of the product ions at $m / z 69,131$, and 181 were monitored as a function of collision energy while keep-

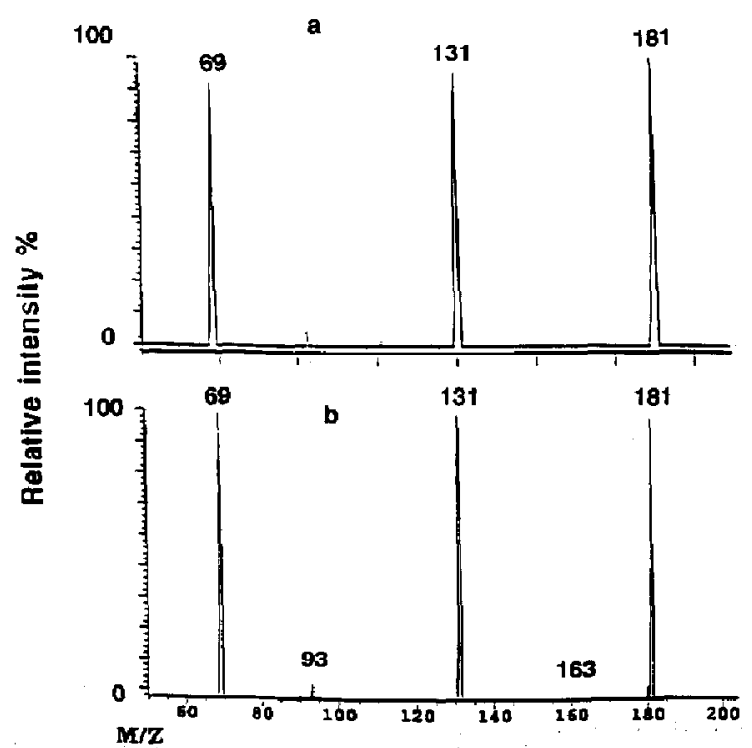

Figure 3. MS/MS spectra of the parent ion at $m / z 231$ using (a) a Finnigan MAT TSQ70 triple quadrupole mass spectrometer (PFTBA) and (b) a VG 70SEQ hybrid geometry instrument (PFK) at the calibration point conditions. ing the target gas pressure constant within the approximate range of $10^{-5}-10^{-6}$ torr (measured on the hybrid instrument), corresponding to a beam attenuation of about $60 \%$. The collision energy at which the intensities of the ions at $m / z 69$ and 181 are equal is defined as the calibration point at that collision gas pressure. The intensity of the product ion at $\mathrm{m} / z 131$ is approximately the same as that of the other ions at this calibration point.

Plots showing the relative intensities of the three product ions at $m / z 69,131$, and 181 at three different indicated target gas pressures are given in Figure 1 for data obtained on the hybrid mass spectrometer. It can be seen that the lab collision energy at which the specified product ion intensities become equal increases with a decrease in collision gas pressure. On a day-to-day basis on the hybrid instrument, with the same indicated collision gas pressure, the reproducibility of the observed collision energy value at the calibration point was $\pm 1-2 \mathrm{eV}$. Figure 2 is the calibration point plot for the "triple quadrupole" mass spectrometer, and shows that the intensities of the ions at $m / z$ 181,131 , and 69 are very nearly equal at the indicated collision energy and pressure. Figure 3 compares the discrete product ion MS/MS spectrum of the ion at $m / z 231$ using the two instruments (the hybrid and the triple quadrupole instrument) under calibration

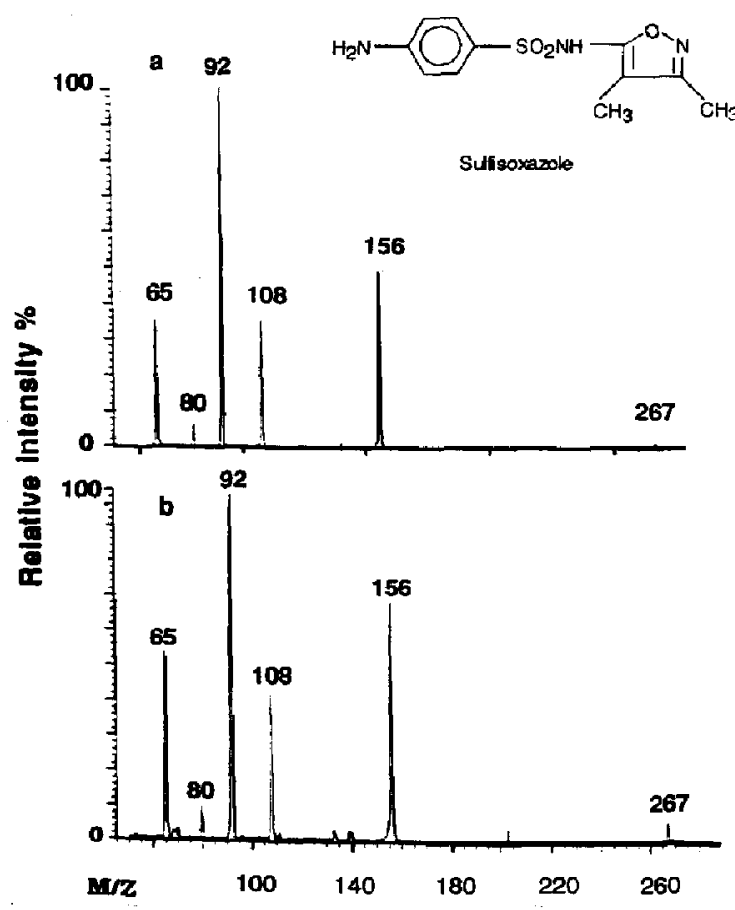

Figure 4. Comparison of MS/MS spectrum obtained at calibration point of sulfisoxazole; parent ion $m / z=267$. The MS $/ \mathrm{MS}$ spectrum from the triple quadrupole mass spectrometer is given in (a), and that from the hybrid geometry instrument in (b). Deviation (defined within manuscript) $=8.4 \%$. 
point conditions. A collision energy of about $50 \mathrm{eV}$ (lab frame) and a measured collision gas pressure of about $10^{-5}$ torr (measured indirectly by an ion gauge outside the collision cell on the hybrid mass spectrometer), or 1 mtorr (as measured on the triple quadrupole mass spectrometer), provides operating conditions near the defined calibration point. Fine tuning $(1-\mathrm{eV}$ increments) of the collision energy is used to reach the defined calibration point. With an increase in collision energy, the relative intensities of higher mass fragments generally decrease while those of lower mass ions increase. Accordingly, as shown in Figure 1 , it can be seen that the intensity of $m / z 69$ increases and the intensity of $m / z 181$ decreases with an increase in collision energy at any particular collision gas pressure. The other fragment ions in the MS/MS spectrum (fragment ions at $m / z 93$ and 163) are of much lower relative intensity, and do not exceed relative intensities of $20 \%$ for collision energies near the calibration value.

We have been repeatedly able to reach conditions in which the product ion EI MS/MS spectrum of the parent ion at $m / z 231$ from PFK or PFTBA is highly reproducible. The general test of usefulness is whether the product ion MS/MS spectra of various compounds, measured at the calibration point, also exhibit good reproducibility when different multiquadrupole instruments are used. We compared product ion EI MS/MS spectra from molecular ions of several differ-

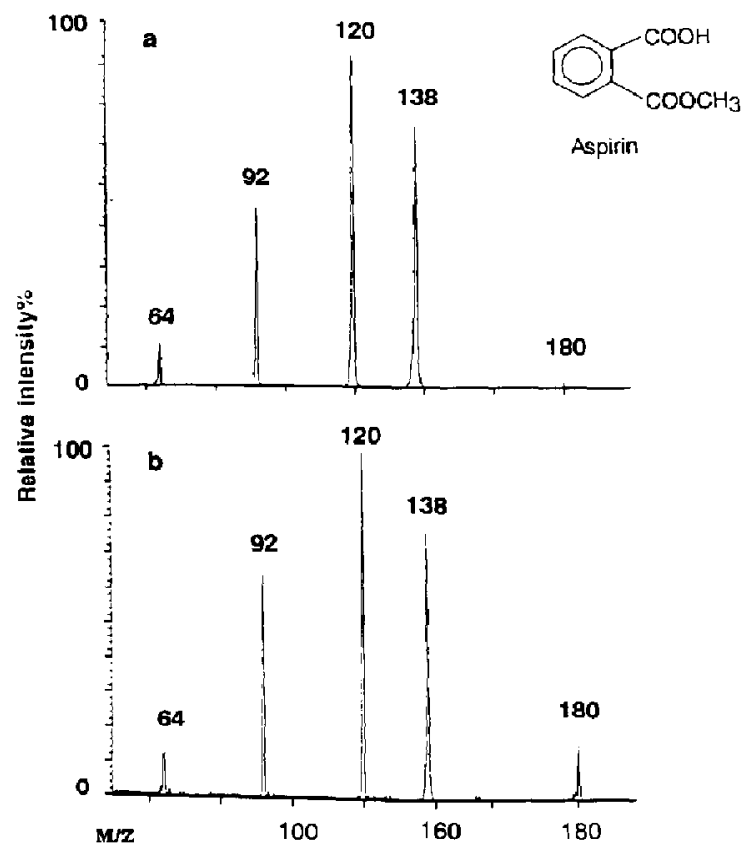

Figure 5. Comparison of MS/MS spectrum of aspirin; parent ion $m / z=180$. The MS $/$ MS spectrum from the triple quadrupole mass spectrometer is given in (a), and that from the hybrid geometry instrument in (b). Deviation (defined within manuscript) $=15.0 \%$. ent classes of compounds using a hybrid mass spectrometer and a triple quadrupole instrument. These comparisons are highlighted in Figures 4-8 for test compounds sulfisoxazole, aspirin, nicotine, caffeine, and the dye acridine orange, respectively. The hybrid instrument MS/MS data shown in Figures 4-8 were measured under conditions shown in Figure $1 \mathrm{~b}$.

Direct comparison of the product ion MS/MS spectrum on a compound-by-compound basis is the best way to assess the reproducibility of the spectra; Figures $4-8$ provide a visual comparison. In general, the tragment ion abundances were similar for each instrument. A quantitative comparison examined the relative intensities for the most abundant fragment ions within the MS/MS spectrum. The fractional intensity contribution of each ion (intensity of that fragment ion divided by the sum of all fragment ion intensities) was calculated for each MS/MS spectrum. The relative percent difference between the measured intensities was calculated as $\left|\left(\operatorname{Int}_{\mathrm{a}}-\operatorname{Int}_{\mathrm{B}}\right)\right| /\left[\left(\operatorname{Int}_{\mathrm{a}}+\operatorname{Int}_{\mathrm{B}}\right) / 2\right] \times$ $100 \%$ for each ion, and then these differences were averaged for the number of fragment ions in the MS/MS spectrum to generate a percent difference. This approach is used to gauge, in general, the ex-

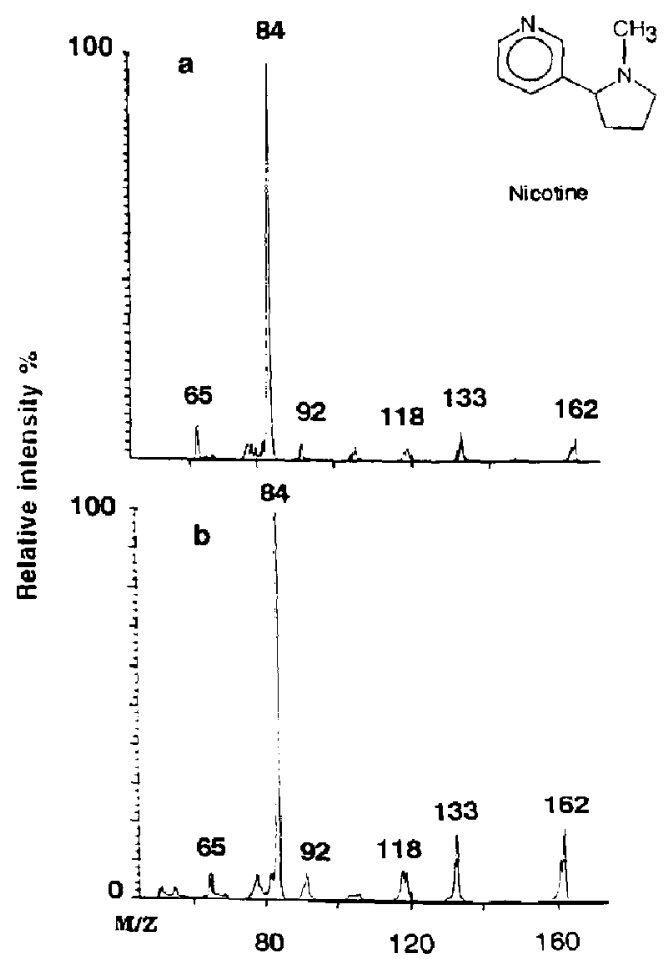

Figure 6. Comparison of MS/MS spectrum of nicotine; parent ion $m / z=162$. The MS $/$ MS spectrum from the triple quadrupole mass spectrometer is given in (a), and that from the hybrid geometry instrument in (b). Deviation (defined within manuscript) $=38.2 \%$. 


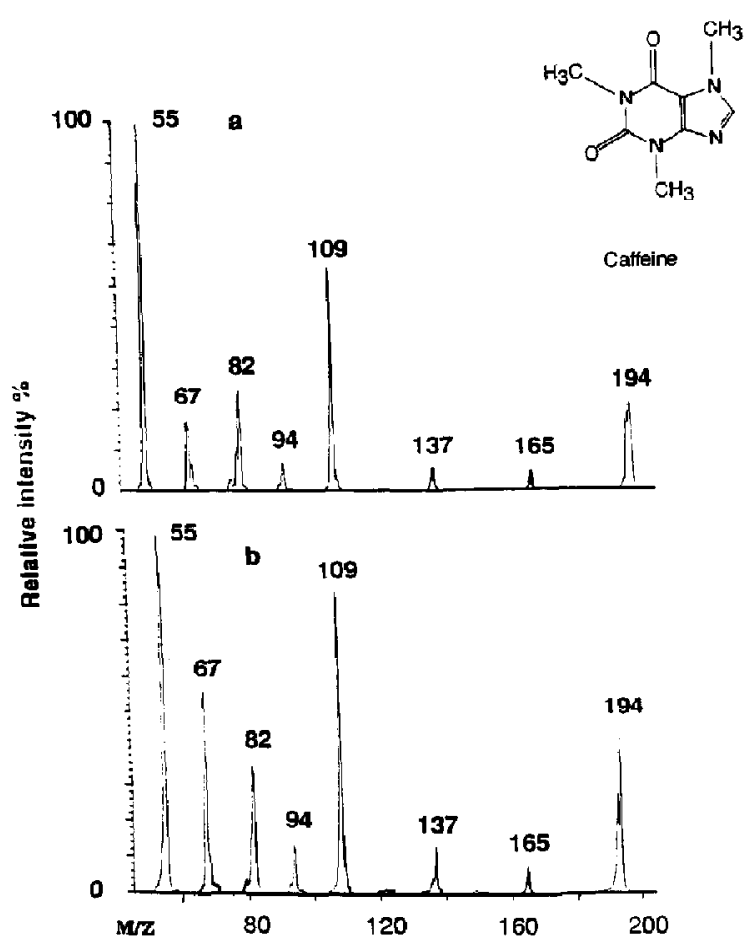

Figure 7. Comparison of MS/MS spectrum of caffeine; parent ion $m / z=194$. The MS/MS spectrum from the triple quadrupole mass spectrometer is given in (a), and that from the hybrid geometry instrument in (b). Deviation (defined within manuscript) $=25.4 \%$

pected range in intensity variations. The range of \pm intensity variation is from $8.4 \%$ for sulfisoxazole to $38.2 \%$ for nicotine (high because of the relatively weak signals for most fragment ions), with an average difference percentage of $21 \%$. Sulfisoxazole was used as a test case to examine the changes in the product ion MS/MS spectra using different calibration points (see Figure 1). Using the same equation shown above to evaluate intensity variations, the variation for the product ions derived from sulfisoxazole from conditions $1 \mathrm{~b}$ to $1 \mathrm{c}$ was $20 \%$.

The ability to compare data repeatedly measured under standard conditions allows subtle trends in the spectra to be identified. Although the product ion MS/MS spectra were overall very similar, there were slight systematic variations. The relative intensities of the parent ions in the MS/MS spectra taken by the triple quadrupole instrument are generally slightly lower than on the hybrid instrument. Indeed, for ideal compounds chosen to illustrate instrument-to-instrument reproducibility, a small change in either direction from the calibration point collision energy significantly changes the relative abundances of product ions; this high dependence is exactly what is desirable in a calibration measurement. We also note that a collision energy range of $50-60 \mathrm{eV}$ (lab frame) provides better

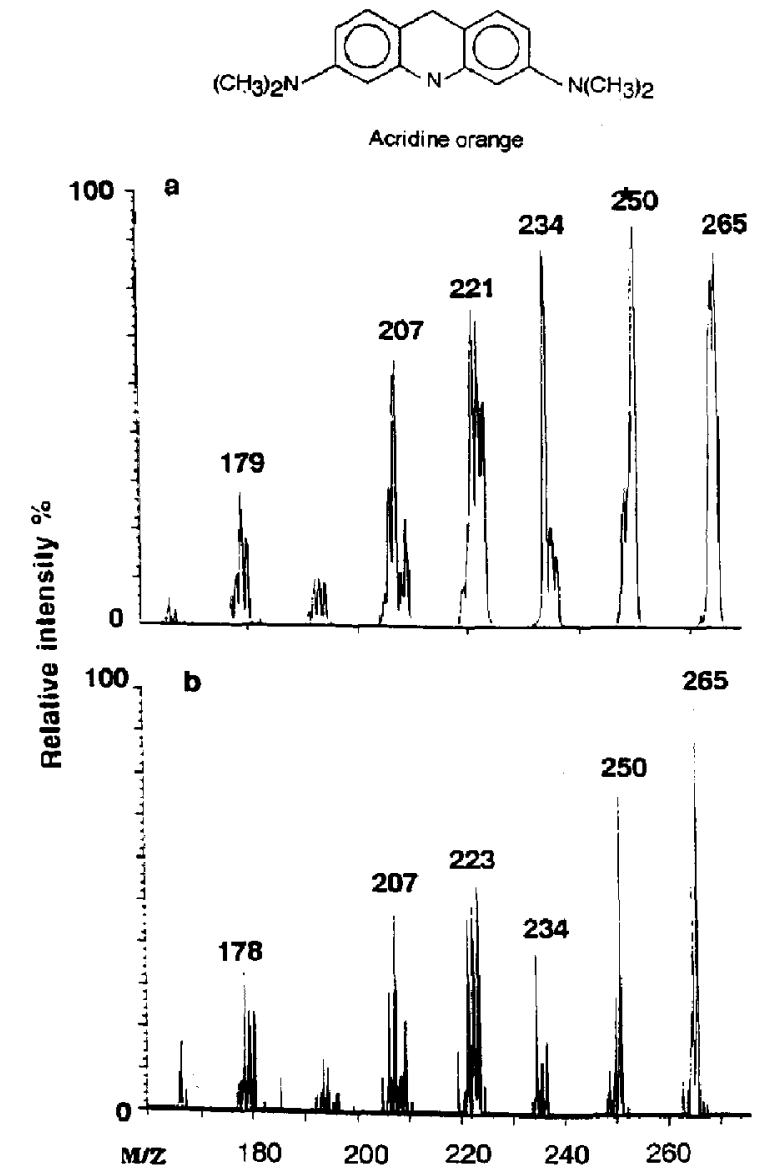

Figure 8. Comparison of MS/MS spectrum of acridine orange: parent ion $m / z=265$. The MS $/ \mathrm{MS}$ spectrum from the triple quadrupole mass spectrometer is given in (a), and that from the hybrid geometry instrument in (b). Deviation (defined within manuscript) $=19.6 \%$.

reproducibility than do the lower collision energy values obtained at the calibration point for higher collision gas pressures, which are harder to stabilize. As expected, small variations in MS/MS spectra may be minimized during an MS/MS experiment by checking the calibration point at regular intervals.

\section{Conclusions}

In this work, a simple procedure for obtaining reproducible MS/MS spectra using EI is described using ions from PFK or PFTBA and easily attainable instrument conditions. EI MS/MS spectra taken at the calibration point using two different instruments show a high degree of similarity for several test compounds. This reproducibility should be sufficient for creation of a common MS/MS database. There is no expectation or claim that the standard conditions used at the cali- 
bration point are ideal for inducing structurally informative dissociation for all classes of compounds, or that the reproducibility of the product ion MS/MS spectra is the equal of that derived from more involved procedures. However, these readily accessible conditions should provide impetus for further instrument-to-instrument comparisons of MS/MS spectra.

\section{Acknowledgments}

Unilever has provided generous support for our research program in tandem mass spectrometry. Kevin L. Duffin participated in some of the early work on this project.

\section{References}

1. Dawson, P. H.; Sun, W.-F. Int. I. Mass Spectrom. Ion Phys. 1983, 55, 155.

2. Dawson, P. H.; Fulford, J. E. Int. I. Mass Spectrom. Ion Phys. 1982, 42, 195.

3. Martinez, R. I.; Dheandhanoo, S. Int. J. Mass Spectrom. Ion Proc. 1986, 74, 241.

4. Martinez, R. I.; Dheandhanoo, S. J. Res. Natl. Bur. Stand. 1987, 92, 229.

5. Martinez, R. I.; Dheandhanoo, S. J. Res. Natl. Inst. Std. Technol. 1989, 94, 281.
6. Martinez, R. I. Rapid Commun. Mass Spectrom. 1991, 5, 245.

7. Martinez, R. I.; Ganguli, B. J. Am. Soc. Mass Spectrom. 1992, 3, 427-444.

8. Mirocha, C. J.; Pawlosky, R. J. Proceedings of the 38th ASMS Conference on Mass Spectrometry and Ailied Topics; Tucson, AZ, June 3-8, 1990.

9. Busch, K. L.; Glish, G. L.; McLuckey, S. A. Mass Spectrometry / Mass Spectrometry: Techniques and Applications of Tandem Mass Spectrometry; VCH: New York, 1988.

10. Mocrery, D. A.; P'eake, D. A.; Gross, M. L. Anal. Chem. 1985, $57,1181$.

11. Naylor, S.; Lamb, J. H. Anal. Chim. Acta 1990, 241, 201.

12. Dawson, P. H. Quadrupole Mass Spectrometry and its Applications; Elsevier: Amsterdam, 1976.

13. Alexander, A. J.; Dyer, E. W.; Boyd, R. K. Rapid Commun. Mass Spectram. 1989, 3, 364.

14. Bartlett, M. G.; Busch, K. L. Anal. Instrum. 1993, 21 (3\& 4), $123-140$.

15. Baeten, W.; Claereboudt, J.; Van Den Heuvel, H.; Claeys, M. Biomed. Environ. Mass Spectrom. 1989, 17, 727.

16. Alexander, A. J; Boyd, R. K. Int. J. Mass Spectrom. Ion Proc. 1989, 90, 211

17. Kim, M. S.; Rabrenovic, M.; Beynon, J. H. Int. J. Mass Spectrom. Ion Proc. 1984, 56, 71.

18. DeLange, W.; Nibbering, N. M, M. Int. J. Mass Spectrom. Ion Proc. $1988,86,28{ }^{7}$.

19. Lamb, J. H.; Naylor, S. Rapid Commun. Mass Spectrom. 1990, 4,421 . 